\title{
EL TRIBUNAL EUROPEO DE DERECHOS HUMANOS ANTE LAS RESTRICCIONES DEL DERECHO A LA ASISTENCIA LETRADA EN LOS PRIMEROS
MOMENTOS DE LA PRIVACIÓN DE LIBERTAD
}

\author{
The European Court of Human Rights' case-law \\ regarding restrictions on the right to prompt \\ legal assistance during police custody
}

\author{
ANTONIO MARTÍNEZ SANTOS \\ Universidad Francisco de Vitoria \\ a.martinez.prof@ufv.es \\ Cómo citar/Citation \\ Martínez Santos, A. (2020). \\ El Tribunal Europeo de Derechos Humanos ante las restricciones del derecho \\ a la asistencia letrada en los primeros momentos de la privación de libertad. \\ Revista Española de Derecho Constitucional, 118, 109-137. \\ doi: https://doi.org/10.18042/cepc/redc.118.04
}

\section{Resumen}

En los últimos años, el Tribunal Europeo de Derechos Humanos se ha pronunciado en repetidas ocasiones sobre la compatibilidad de las restricciones del derecho a un abogado en dependencias policiales con los arts. 6.1 y 6.3 c) del Convenio Europeo de Derechos Humanos. En la sentencia del asunto Salduz c. Turquía, el Tribunal dictaminó que la privación injustificada del acceso a un abogado constituye por sí sola una violación del Convenio, con independencia de su impacto posterior en la causa. Sin embargo, pronunciamientos ulteriores del TEDH han venido a arrojar dudas sobre el verdadero alcance de la transformación que parecía derivarse de Salduz. Este trabajo analiza la evolución de la jurisprudencia de Estrasburgo sobre las restricciones del derecho a la asistencia letrada en los primeros momentos de la privación de libertad. 


\title{
Palabras clave
}

Asistencia letrada; garantías procesales; proceso justo; derechos de defensa; custodia policial; Tribunal Europeo de Derechos Humanos.

\begin{abstract}
Over the past few years, the European Court of Human Rights has been confronted repeatedly with the question of whether restrictions on the right to legal assistance during police custody constitute a breach of Articles 6.1 and $6.3 \mathrm{c}$ ) of the European Convention on Human Rights. In its landmark decision in Salduz v Turkey, the Court seemed to hold that any unjustified denial of access to counsel during police interrogation entails in itself a violation of the Convention, irrespective of its subsequent impact on the proceedings. However, later judgments of the ECtHR cast significant doubts as to the real scope of the transformation that Salduz seemed to imply. This paper analyses the development of Strasbourg's case law concerning restrictions on the right of access to a lawyer in the early stages of pre-trial arrest.
\end{abstract}

\section{Keywords}

Legal assistance; procedural safeguards; fair trial; rights of defence; police custody; European Court of Human Rights. 
I. INTRODUCCIÓN. II. LA APROXIMACIÓN INICIAL DEL TEDH: LA PRESERVACIÓN DEL DERECHO DE DEFENSA COMO RATIO DE LA NECESIDAD DE ASEGURAR LA ASISTENCIA LETRADA EN LAS DILIGENCIAS POLICIALES. III. ¿̇EL NACIMIENTO DE UNA REGLA DE EXCLUSIÓN A PARTIR DEL ARTÍCULO 6.3 DEL CONVENIO? LA SENTENCIA DE LA GRAN SALA EN EL ASUNTO SALDUZ C. TURQUÍA. IV. LA RELATIVIZACIÓN POSTERIOR DE LOS CRITERIOS FIJADOS EN SALDUZ Y EL CAMBIO DE PERSPECTIVA EN CUANTO AL IMPACTO DE LA RESTRICCIÓN DEL DERECHO EN LA CAUSA PENAL: ASUNTO IBRAHIM Y OTROS C. REINO UNIDO. V. LA APLICACIÓN DEL ESTÁNDAR DE LA EQUIDAD GLOBAL DE LA CAUSA MÁS ALLÁ DE LA PERSECUCIÓN DE LOS ILÍCITOS MÁS GRAVES: ASUNTO SIMEONOVI C. BULGARIA. VI. LA CONSOLIDACIÓN DE LA LÍNEA JURISPRUDENCIAL POST-IBRAHIM: ASUNTO BEUZE C. BÉLGICA. VII. CONSIDERACIONES FINALES. BIBLIOGRAFIA.

\section{INTRODUCCIÓN}

La jurisprudencia del Tribunal Europeo de Derechos Humanos sobre las restricciones del derecho a la asistencia letrada en los estadios preliminares de la investigación penal ( $\mathrm{y}$, especialmente, en los primeros momentos de la detención policial) ha conocido importantes fluctuaciones en las últimas dos décadas. El primer punto de inflexión relevante vino marcado por la sentencia de la Gran Sala en el asunto John Murray c. Reino Unido, que inauguró una línea de argumentación que se desarrolló durante años y culminó en la sentencia del asunto Salduz c. Turquia.

En esta última resolución, que tuvo tanta trascendencia como para inducir cambios legislativos en algunos Estados miembros del Consejo de Europa - e influir de forma decisiva en los trabajos preparatorios de la Directiva 2013/48/UE—, el TEDH sentó una serie de principios que configuraron lo que se dio en llamar el «test Salduz», que había de servir a modo de instrumento analítico para determinar cuándo una privación del derecho de acceso temprano a un abogado puede considerarse ilegítima desde el punto de vista de las exigencias del Convenio Europeo de Derechos Humanos.

1 Este trabajo es resultado de una estancia en la Universidad de Bolonia, cofinanciada por la Universidad Francisco de Vitoria y el proyecto «El proceso penal en la era digital. Retos para los derechos humanos en el espacio europeo y transnacional» (FEI-EU 19/11). 
Sin embargo, en resoluciones posteriores el Tribunal de Estrasburgo alteró el enfoque y rectificó el criterio adoptado en Salduz, orillando el control de compatibilidad con el art. 6.3 c) del CEDH que había introducido aquella sentencia y regresando a la aproximación jurisprudencial más primitiva, consistente en efectuar un indiferenciado «examen de conjunto» de las actuaciones procesales, a efectos de valorar si la restricción del acceso a un abogado por parte de las autoridades fue o no en detrimento de la equidad global de la causa penal considerada como un todo (lo que en la jurisprudencia actual del Tribunal se conoce como overall fairness test).

A día de hoy, hay razones para pensar que la cuestión no ha quedado definitivamente zanjada. Ciertamente, el sugestivo horizonte que se abrió con la sentencia del asunto Salduz parece haberse disipado por completo, al menos por el momento. Sin embargo, ahora mismo es difícil predecir hacia dónde se dirigirá en el futuro la jurisprudencia de Estrasburgo en lo que a las garantías del art. 6.3 del CEDH se refiere: si lograrán conservar una individualidad y sustantividad propias o si, por el contrario, acabarán viéndose todas confundidas o embebidas definitivamente en ese «examen de conjunto» al que, según parece, el Tribunal ha decidido reconducir las denuncias relacionadas con posibles vulneraciones de los distintos apartados de ese trascendental precepto del Convenio.

\section{LA APROXIMACIÓN INICIAL DEL TEDH: LA PRESERVACIÓN DEL DERECHO DE DEFENSA COMO RATIO DE LA NECESIDAD DE ASEGURAR LA ASISTENCIA LETRADA EN LAS DILIGENCIAS POLICIALES}

Como se acaba de decir, el primer hito importante en la jurisprudencia de Estrasburgo sobre el derecho a un abogado en dependencias policiales fue la sentencia de la Gran Sala en el asunto John Murray c. Reino Unido, que se dictó en $1996^{2}$. Ya desde un tiempo antes, concretamente desde la sentencia del asunto Imbrioscia c. Suiza ${ }^{3}$, el Tribunal había empezado a afirmar que, en materia penal, las garantías de los apartados 6.1 y 6.3 letra c) del Convenio -que son, nótese bien, garantías del proceso justo - deben operar aunque la causa judicial no esté iniciada en sentido estricto; pero que la concreta forma en que lo hagan dependerá de las características particulares de la diligencia a la que se someta al detenido, así como de las circunstancias del caso ${ }^{4}$.

\footnotetext{
STEDH (GS) de 9 de febrero de 1996, asunto John Murray c. Reino Unido.

STEDH de 24 de noviembre de 1993, asunto Imbrioscia $c$. Suiza.

$4 \quad$ STEDH Imbrioscia, $\$ \$$ 36-38. En aquel caso, la decisión final del TEDH fue desestimatoria; pero la sentencia contó con tres votos particulares de los jueces Pettiti, De
} 
En la sentencia John Murray el Tribunal dio un paso más. Por un lado, desarrolló por extenso la fundamentación del criterio expresado en Imbrioscia, explicando que, en el fondo, la razón última de que algunas de las garantías del art. 6 del CEDH deban proyectarse sobre las actuaciones policiales previas al proceso (singularmente, sobre el interrogatorio policial) se encuentra en la necesidad de subvenir prospectivamente a la preservación del derecho de defensa. Es decir, allí donde la ley nacional permite anudar consecuencias al comportamiento del detenido en dependencias policiales en el plano probatorio (consecuencias que pueden incidir, a menudo de manera determinante, en las posibilidades ulteriores de defensa dentro del proceso), el art. 6 del CEDH exigiría con carácter general que se le asegurase el acceso a un abogado ya en ese primer momento5. Por otro lado, consideró que una actuación policial ajustada a la legalidad vigente en el país en el que se produce puede, pese a todo, entrañar una vulneración de los derechos reconocidos en ese precepto ${ }^{6}$.

En efecto, la legislación vigente en Irlanda del Norte en el momento de producirse los hechos que dieron lugar a la sentencia del asunto John Murray permitía a la policía, en determinadas circunstancias, restringir válidamente el acceso del sospechoso a un abogado por un tiempo de hasta cuarenta y ocho horas desde la detención. Y eso fue precisamente lo que sucedió: detenido el sr. Murray como sospechoso de terrorismo, el superintendente de policía acordó retrasar la asistencia letrada por el plazo de cuarenta y ocho horas fijado en la ley. En ese tiempo, el sr. Murray fue interrogado doce veces, sumando todos los interrogatorios un total de veintiuna horas. En todos ellos el detenido se negó a declarar; negativa que, según la ley entonces en vigor, permitía al juez penal realizar inferencias respecto de su participación en los hechos. El TEDH consideró que, tomadas en su conjunto, las circunstancias descritas abocaban al detenido a un auténtico dilema. O bien guardaba silencio, y entonces ese silencio podía ser utilizado como indicio contra él, o bien respondía a las preguntas de los agentes de policía, en cuyo caso podía estar condicionando gravemente su defensa en el proceso posterior, probablemente sin ser consciente de ello (y, por otra parte, tampoco se libraba de posibles inferencias lógicas en su contra, en función de la verosimilitud que el juez decidiera atribuir a lo declarado y de la valoración que hiciese de sus silencios u omisiones). Por estos motivos, la sentencia John Murray terminó

Meyer y Lopes Rocha, que, en cierto modo, sirvieron de base para el desarrollo jurisprudencial posterior.

5 STEDH John Murray, $\$ 63$.

6 STEDH John Murray, \$\$ 65-66. 
dictaminando que negar el acceso a un abogado durante las primeras cuarenta y ocho horas de interrogatorios policiales, en un contexto legal en el que, haga lo que haga el detenido, el derecho de defensa en un eventual proceso puede verse comprometido de forma irreparable, es incompatible con el art. 6 del Convenio, sean cuales sean las razones invocadas por las autoridades para justificar la limitación?

Importa aclarar que la sentencia John Murray no llegó a decir que el derecho a la asistencia letrada en dependencias policiales (que no está expresamente reconocido como tal en el Convenio) sea un derecho absoluto e incondicionado; ni siquiera cuando de interrogatorios por parte de los agentes se trata. Al contrario, la sentencia afirmó expresamente que se trata de un derecho que puede verse limitado si hay «razones fundadas» (good cause; raisons valables) para ello; y echó mano del tradicional criterio conforme al cual, en cada caso que se presente, el examen a efectos de discernir si una determinada restricción impuesta por las autoridades de persecución penal ha infringido las garantías mínimas del art. 6 del CEDH [en este supuesto particular, la del apartado $3 .^{\circ}$, letra c)] debe consistir en ponderar si, atendido el procedimiento en su conjunto, puede considerarse que la restricción en cuestión supuso un menoscabo del derecho a un juicio justo ${ }^{8}$.

\section{III. ¿̇EL NACIMIENTO DE UNA REGLA DE EXCLUSIÓN A PARTIR DEL ARTÍCULO 6.3 DEL CONVENIO? LA SENTENCIA DE LA GRAN SALA EN EL ASUNTO SALDUZ C. TURQUÍA}

Más importante si cabe que la sentencia John Murray fue la sentencia de la Gran Sala de 2008 en el asunto Salduz c. Turquía', que estimó por unanimidad la reclamación presentada por un ciudadano turco que había sido condenado penalmente sobre la base de manifestaciones realizadas en un interrogatorio policial practicado sin abogado.

En esencia, los hechos a los que se refirió la sentencia Salduz son los siguientes: a finales de 2001, el sr. Yusuf Salduz, que entonces era menor de edad, fue detenido por la policía turca, que le atribuía la participación en una manifestación ilegal de apoyo a la organización terrorista Partido

STEDH John Murray, $\$ 66$, in fine.

8 STEDH John Murray, $\$$ 63, in fine. También en relación con la garantía del art. 6.3 c) del Convenio, véanse las SSTEDH de 6 de junio de 2000, asunto Magee c. Reino

Unido, $\$ 41$, y de 16 de octubre de 2001, asunto Brennan c. Reino Unido, $\$ 45$.

9 STEDH (GS) de 27 de noviembre de 2008, asunto Salduz c. Turquía. 
de los Trabajadores del Kurdistán (PKK). El sospechoso fue interrogado sucesivamente por la policía, por el representante del Ministerio Público y por el juez instructor del caso. En ninguno de los interrogatorios estuvo asistido de letrado, ni antes, ni durante, ni después de la diligencia. Fue solamente después de los interrogatorios, una vez acordada la prisión provisional por el juez de instrucción, que se le permitió entrevistarse con su abogado. Celebrado el juicio, el acusado resultó condenado a cuatro años y seis meses de prisión, que quedaron reducidos a dos años y seis meses por su condición de menor en el momento de producirse los hechos por los que había sido juzgado. En la sentencia de instancia, que fue confirmada en apelación, el tribunal utilizó como prueba de cargo las declaraciones autoincriminatorias que el acusado había efectuado en sede policial (y que luego habían sido retractadas ante el fiscal, ante el juez de instrucción y en el propio acto del juicio oral, aduciendo que se habían producido bajo coacción).

$\mathrm{Al}$ abordar el análisis del caso, la Gran Sala empezó recordando la jurisprudencia anterior sobre la asistencia letrada en sede policial (sentencias Imbrioscia y John Murray), justificándola sobre la base de tres argumentos: protege al sospechoso frente a posibles abusos, ayuda a evitar las decisiones injustas y contribuye a la consecución de los fines del art. 6 del CEDH, especialmente en lo que se refiere al establecimiento de una cierta «igualdad de armas» entre las autoridades de persecución penal y la persona contra la que se dirigen las actuaciones. A ojos del Tribunal, en materia penal la fase de investigación tiene una importancia decisiva, pues muchas veces el material obtenido en ella proporciona el marco en el que los hechos investigados son considerados en el juicio. Al mismo tiempo, el sospechoso se encontraría en una posición particularmente vulnerable en esa fase desde el punto de vista de las garantías de un proceso justo; efecto que se puede ver agravado, además, por la complejidad técnica de la legislación procesal penal, especialmente en lo que a la localización, recogida y utilización de fuentes de prueba dentro del proceso se refiere. Según el razonamiento de la sentencia Salduz, esta «vulnerabilidad» del sospechoso durante la investigación policial preliminar solo puede verse adecuadamente compensada mediante la asistencia de un abogado, cuyo cometido principal es velar por que se respete en todo momento el derecho del sospechoso a no autoincriminarse. Para la Gran Sala, el llamado «privilegio de la no-autoincriminación» supone (entre otras cosas) que las autoridades de persecución penal deben levantar la carga de la prueba respecto de los hechos en los que se basa su pretensión acusatoria sin recurrir a materiales o informaciones obtenidos mediante coacción, o contra la voluntad del sujeto investigado. El acceso temprano a un abogado es, de este modo, una 
parte esencial de las salvaguardias procesales que rodean al derecho a no declarar contra sí mismo ${ }^{10}$.

Por todos estos motivos, la sentencia Salduz concluye que, para que el derecho al juicio justo sea "práctico y efectivo» en el sentido exigido por la jurisprudencia de Estrasburgo, el art. 6.1 del CEDH obliga, como regla general, a que el acceso al abogado se produzca ya desde el primer interrogatorio del sospechoso por parte de la policía ${ }^{11}$, pues el derecho de defensa en el proceso se ve comprometido de forma irremediable cuando una condena penal se sustenta en declaraciones autoincriminatorias prestadas ante los agentes de policía sin haber tenido acceso a la asistencia de letrado ${ }^{12}$. Esta regla conocería una única excepción, y es que, a la luz de las circunstancias del caso, se demuestre que concurrían "razones imperiosas» (compelling reasons; raisons impérieuses) para restringir el acceso del detenido a un abogado. Y aun en ese supuesto, es preciso comprobar que la restricción impuesta no repercutió negativamente en el resto de garantías que conforman el derecho a un proceso justo, pues, de lo contrario, el art. 6 del $\mathrm{CEDH}$ se habría visto vulnerado de todos modos ${ }^{13}$.

De estas consideraciones nació el conocido como «test Salduz», un instrumento de análisis concebido para facilitar la tarea de dilucidar cuándo las restricciones impuestas al derecho a la asistencia letrada temprana por parte de la policía pueden reputarse lesivas del derecho a un proceso justo del art. 6 del $\mathrm{CEDH}$. Dicho test consta de dos fases. En un primer momento, se evalúa si puede entenderse que había «razones imperiosas» para limitar o retrasar el acceso del detenido a un abogado en sede policial. En caso afirmativo (esto es, de verificarse que efectivamente esas razones existieron), la segunda fase consiste en comprobar si la limitación del derecho a la asistencia letrada en comisaría tuvo consecuencias procesales que repercutieron de alguna forma en la decisión final; y si, por ese motivo, se vio afectado el adecuado ejercicio del derecho de defensa en la causa (pues bien puede ser que no haya habido afectación desde ese punto de vista, o que sí la haya habido pero sea desdeñable o se

10 STEDH Salduz, $\$ 54$. Para una exposición completa del tratamiento de este derecho en la jurisprudencia de Estrasburgo, vid. Ormazábal Sánchez (2015: 130-171).

11 En la sentencia Salduz no se llegó a afirmar que el derecho a la asistencia letrada nazca en el momento de pasar a estar bajo custodia policial (sí se dijo, en cambio, en uno de los votos particulares). Esta extensión se produciría más adelante en la STEDH de 13 de octubre de 2009, asunto Dayanan c. Turquía.

12 STEDH Salduz, $\$ \$ 55$, in fine.

13 STEDH Salduz, $\$ \$ 50-55$. Debe notarse por añadidura que la sentencia reconoce la posibilidad de renunciar a las garantías del art. 6 del CEDH. Al respecto, véanse las afirmaciones contenidas en el $\$ 59$. 
haya visto compensada o «balanceada» de otro modo, en cuyo caso no se habría vulnerado el derecho al proceso justo del art. 6 del CEDH). Es importante subrayar que en Salduz el TEDH no quiso abrogar ni suprimir el overall fairness test, sino solamente posponerlo. Habría de entrar en juego una vez constatada la concurrencia de razones imperiosas para privar del acceso temprano a un abogado. En definitiva, del razonamiento de la sentencia se desprende que la privación de asistencia letrada podría acabar resultando ilegítima desde el punto de vista del derecho a un proceso justo, aun estando prevista legalmente $y$ aun concurriendo «razones imperiosas» para que se produjese.

Aplicado el examen que se acaba de describir sumariamente el caso concreto del sr. Salduz, la Sala constató que en ningún momento se habían aducido "razones imperiosas» para limitar el acceso a un abogado en los primeros momentos de la detención. Por eso entendió que, en aquel supuesto y por ese solo motivo, había habido ya vulneración del art. 6.3, letra c) del Convenio. Sin embargo, en lugar de detenerse ahí (que tal vez habría sido lo más coherente con el planteamiento desarrollado en la fundamentación jurídica de la sentencia), entró a valorar también la incidencia de la restricción en las actuaciones posteriores, llegando a la conclusión de que el derecho de defensa del sr. Salduz en la causa penal seguida contra él se había visto condicionado negativamente por la actuación inicial de las autoridades, y de forma además irreparable, porque las declaraciones prestadas sin asistencia letrada no habían quedado fuera del proceso, sino que se habían utilizado directamente para sustentar la condena ${ }^{14}$.

Los principios sentados con ocasión del asunto Salduz tuvieron gran acogida doctrinal y sirvieron de base a las diferentes secciones del TEDH para abordar el problema en multitud de casos posteriores. Su alcance se proyectó incluso más allá de la jurisprudencia de Estrasburgo, ya que varios Estados miembros del Consejo de Europa introdujeron cambios en sus legislaciones internas para adaptarlas a los nuevos criterios, garantizando el acceso del detenido a un abogado en el interrogatorio policial allí donde, tradicionalmente, esa posibilidad ni siquiera se contemplaba ${ }^{15}$. La misma Comisión Europea

14 STEDH Salduz, \$\$ 57-58 y 62. Aunque el Tribunal no mencionó en ningún momento por su nombre el caso Miranda c. Arizona, parece claro que Salduz se inspira en aquel precedente de la jurisprudencia de la Corte Suprema de Estados Unidos (que sí había sido citado en dos de los votos particulares a la sentencia del asunto John Murray). Sobre los paralelismos entre Miranda y Salduz, vid. Weisselberg (2017: 1252-1255).

15 Es el caso, señaladamente, de Irlanda, Francia, Bélgica y Holanda. Al respecto, vid. Giannoulopoulos (2016: 112-120), Mols (2017: 302-304) y, para los ordenamientos francés y belga, Beernaert et al. (2012: 249-261). 
basó su propuesta de Directiva sobre el derecho de acceso a un abogado en la fundamentación jurídica de la sentencia Salduz ${ }^{16}$.

No faltó quien interpretara que el TEDH en Salduz había creado una nueva regla de exclusión probatoria, que afectaría a cualesquiera declaraciones obtenidas por las autoridades tras haber privado injustificadamente al sujeto interrogado de la asistencia de un abogado ${ }^{17}$. Así se desprendería, además, de otros pronunciamientos que se sucedieron en los años inmediatamente posteriores a Salduz ${ }^{18}$.

Desde luego, ello habría supuesto un giro de enorme trascendencia, porque hasta ese momento el Tribunal de Estrasburgo se había abstenido siempre de enunciar proposiciones o dictámenes de alcance general acerca de las condiciones para la recepción de los resultados de la investigación penal en la fase de juicio, de conformidad con la conocida pauta según la cual el establecimiento de las reglas sobre la admisibilidad de la prueba y su valoración por los tribunales competen primordialmente al derecho interno de los Estados signatarios del Convenio ${ }^{19}$. El TEDH reconocía hasta entonces una única excepción, la de las informaciones obtenidas a partir de torturas o tratos inhumanos o degradantes (art. 3 del CEDH), cuya recepción y utilización en la causa vulneraría siempre el derecho a un proceso justo, con independencia de su veracidad intrínseca y de si sirvieron efectivamente o no como fundamento de la condena ${ }^{20}$.

16 Véase la Propuesta de Directiva de 8 de junio de 2011 (Ref. COM[2011] 326 final). En general, sobre la directiva y sus antecedentes, vid. Cras (2014), Arangüena Fanego (2014), Jimeno Bulnes (2014) y Bachmaier Winter (2015). Sobre la influencia de Salduz en los trabajos prelegislativos de la directiva, vid. Anagnostopoulos (2014: 6-18).

17 Véase, por ejemplo, Ölçer (2013: 390), quien la califica de regla de exclusión cuasi absoluta (nearly absolute). En un sentido parecido, vid. Celiksoy (2018: 244).

18 Vid., por ejemplo, las SSTEDH de 11 de diciembre de 2008, asunto Panovits c. Chipre, $\$ 66$; de 31 de marzo de 2009, asunto Ptonka c. Polonia, $\$ \$ 35$ y 39-41, y de 24 de septiembre de 2009, asunto Pishchalnikov c. Rusia, $\$ 81$.

19 Vid., por todas las SSTDEH de 12 de julio de 1988, asunto Schenk c. Suiza, $\$ 46$; de 12 de mayo de 2000, asunto Khan c. Reino Unido, $\$ 34$; de 25 de septiembre de 2001, asunto P. G. y J. H. c. Reino Unido, $\$ 76$; de 5 de noviembre de 2002, asunto Allan c. Reino Unido, $\$ 42$; de 1 de junio de 2010, asunto Gäfgen c. Alemania, $\$ \$ 162-163$, y de 15 de diciembre de 2011, asunto Al-Khawaja y Tahery c. Reino Unido, $\$ 118$.

20 Vid. la STEDH de 1 de junio de 2010, asunto Gäfgen c. Alemania, $\$ 166$. Paradójicamente, aquella decisión fue, sin embargo, desestimatoria en lo que a la vulneración del art. 6 del CEDH se refiere, por acogerse el Tribunal a una versión de lo que, entre nosotros, se conoce como la «desconexión de antijuridicidad». Véase al respecto el 


\section{LA RELATIVIZACIÓN POSTERIOR DE LOS CRITERIOS FIJADOS EN SALDUZ Y EL CAMBIO DE PERSPECTIVA EN CUANTO AL IMPACTO DE LA RESTRICCIÓN DEL DERECHO EN LA CAUSA PENAL: ASUNTO IBRAHIM Y OTROS C. REINO UNIDO}

Sea como fuere, lo cierto es que muy pronto empezaron a surgir fisuras en la jurisprudencia de la Gran Sala en torno a los criterios introducidos en Salduz. La primera resolución donde se puede apreciar esta tendencia es la sentencia del asunto Dvorski c. Croacia ${ }^{21}$, que elaboró un segundo test, menos exigente que el de la sentencia Salduz, para los casos en los que la restricción impuesta por la policía se refiere no ya a la posibilidad de acceder de forma temprana a un abogado, sino a la libertad del detenido a la hora de elegirlo $^{22}$. Para la sentencia Dvorski, privar al detenido de la posibilidad de escoger un letrado de su confianza en dependencias policiales no equivale a privarle del derecho a la asistencia letrada tout court. La primera privación revestiría «menos gravedad» que la segunda. Por eso, en Dvorski el Tribunal consideró que debía aplicar un estándar más relajado, consistente en determinar si en el caso se daban «motivos relevantes y suficientes» (relevant and sufficient grounds; motifs pertinents et suffisants) para impedir al detenido la designación del abogado preferido por él. De no concurrir esos motivos (adviértase la inversión del planteamiento con respecto a Salduz), habría entonces que valorar la equidad global (overall fairness) del procedimiento en su conjunto, atendiendo a una serie de circunstancias que la sentencia enumera a título meramente ejemplificativo (la eficacia de la asistencia prestada por el letrado designado de oficio, la utilización posterior en el proceso de las declaraciones realizadas ante los agentes, si se respetó el derecho a no declarar contra sí mismo, etc. $)^{23}$.

Con todo, es solo a partir de la controvertida sentencia de la Gran Sala en el asunto Ibrahim y otros $c$. Reino Unido ${ }^{24}$, concerniente a cuatro terroristas islámicos condenados por una serie de atentados frustrados en Londres, que se observan ya con nitidez un cierto distanciamiento y una cierta relativización del alcance del test Salduz, al hilo de una pretendida «clarificación» de

$\$ 180$ de la sentencia. Para un comentario crítico de la sentencia Gäfgen, vid. Maffei y Sonenshein (2012).

21 STEDH (GS) de 20 de octubre de 2015, asunto Dvorski c. Croacia.

22 STEDH Dvorski, $\$ 81$.

23 STEDH Dvorski, $\$ 82$.

24 STEDH (GS) de 13 de septiembre de 2016, asunto Ibrahim y otros c. Reino Unido. 
sus dos etapas y la relación entre ambas ${ }^{25}$. Esta relativización no obedeció sin más a las peculiaridades sustantivas específicas del asunto resuelto en aquella demanda (como en un principio parecía, al tratarse de delitos de terrorismo particularmente graves), sino que después ha tenido confirmación en sentencias de la Gran Sala referidas a otras clases de supuestos; singularmente, en las sentencias de los asuntos Simeonovi c. Bulgaria y Beuze c. Bélgica, a las que se hará referencia más adelante.

La sentencia Ibrahim empieza recordando una vez más las proclamaciones anteriores del TEDH sobre este punto: el pronto acceso a un abogado es una de las exigencias fundamentales del proceso justo, constituye un importante "contrapeso" a la vulnerabilidad de los sujetos que se encuentran bajo custodia policial, proporciona una protección fundamental contra posibles coacciones y malos tratos policiales y sirve a la consecución de los objetivos del art. 6 del $\mathrm{CEDH}$, particularmente en lo que se refiere al equilibrio de posiciones procesales entre el investigado y las autoridades de persecución penal ${ }^{26}$. Las garantías del art. 6 del Convenio deben entrar en juego tan pronto como existan "cargos penales» contra un sujeto. A estos efectos, el de "cargos penales» es un concepto autónomo, cuyo contenido corresponde al propio TEDH concretar. Básicamente, existirían cargos penales en el sentido definido por la jurisprudencia de Estrasburgo no solo desde el momento de la notificación oficial a un sujeto de que se ha producido una alegación de relevancia penal contra él; sino, en todo caso, desde que su situación se haya visto «sustancialmente afectada» por actuaciones o medidas adoptadas por las autoridades en relación con su persona, aunque sea en virtud de meras sospechas ${ }^{27}$.

Ahora bien, como admite la jurisprudencia previa, hay situaciones en las que, excepcionalmente, el derecho de asistencia letrada en los primeros momentos de privación de libertad se puede retrasar o limitar de manera temporal ${ }^{28}$. Concretamente, se trata de casos en los que existen «razones imperiosas» para restringir el derecho de acceso inmediato a un abogado; extremo que, al no estar definido con exactitud en la jurisprudencia del Tribunal, solamente se puede determinar

25 STEDH Ibrahim, $\$ 257$. Dada su extensión, no es posible ofrecer aquí un análisis exhaustivo de la sentencia Ibrahim. Baste indicar que no todas las demandas corrieron la misma suerte, porque la situación del cuarto denunciante era diferente de la de los tres primeros. Para un tratamiento detallado, vid. Buric (2018). STEDH Ibrahim, $\$ 255$ (con cita de otras resoluciones anteriores).

27 STEDH Ibrahim, $\$ \$ 249$ y 253.

28 STEDH Ibrahim, $\$ 256$. El Tribunal invoca expresamente las sentencias de los asuntos John Murray c. Reino Unido, Magee c. Reino Unido, Brennan c. Reino Unido y Salduz c. Turquía. 
caso por caso, atendiendo en primer lugar a lo prescrito en la normativa procesal de carácter interno ${ }^{29}$.

A continuación, el TEDH se pregunta si la ausencia de «razones imperiosas" para restringir el derecho de acceso inmediato a un abogado (o la falta de justificación de su concurrencia por parte del Estado demandado) basta por sí sola para entender que se ha vulnerado el art. 6 del CEDH, como parecería deducirse de la sentencia Salduz (y como afirmaban los demandantes). Para la sentencia Ibrahim la respuesta es negativa, porque las garantías del art. 6 del CEDH no son "fines en sí mismos", ni deben ser interpretadas de forma que supongan «dificultades desproporcionadas» para la labor de las autoridades ${ }^{30}$. Por otro lado, el hecho de que la propia sentencia Salduz no se limitara a constatar la ausencia de razones imperiosas en el caso que abordó, sino que decidiera entrar a analizar también el impacto posterior en el proceso de la irregularidad inicial detectada, de alguna manera evidenciaría que el criterio de las razones imperiosas es un factor más que debe ser tenido en cuenta, pero que no tiene carácter decisivo ni determinante ${ }^{31}$.

En consecuencia, la sentencia Ibrahim considera que el punto crucial no es tanto la existencia o inexistencia de motivos de peso para restringir el acceso al abogado, como el impacto que esta decisión haya podido tener en la equidad del procedimiento en su conjunto ${ }^{32}$. La única relevancia real que tendría el hecho de que no se hubieran dado las «imperiosas razones» a que se refiere la sentencia Salduz sería la de arrojar sobre el Estado demandado la carga de demostrar de manera convincente que la equidad global del proceso no se vio comprometida con la limitación del derecho a la asistencia letrada en comisaría ${ }^{33}$.

La sentencia Ibrahim rechaza categóricamente que del art. 6 del CEDH y la jurisprudencia que lo interpreta pueda derivarse la existencia de una regla de exclusión implícita, que prohíba tener en cuenta sin más las declaraciones prestadas sin asistencia de abogado ante las autoridades de persecución penal ${ }^{34}$. Fuera de los casos de torturas, para la sentencia el objetivo principal del análisis a la luz del Convenio debería consistir en esclarecer si la equidad o la justicia global de la causa considerada como un todo se ha visto comprometida para el acusado o no, atendidas las circunstancias en que se desarrollaron

\footnotetext{
STEDH Ibrahim, $\$ 258$.

STEDH Ibrahim, \$\$252 y 262.

STEDH Ibrahim, $\$ 260$.

STEDH Ibrahim, $\$ 262$.

STEDH Ibrahim, $\$ 265$.

34 STEDH Ibrahim, $\$ 261$.
} 
las diligencias policiales, la información obtenida en ellas y el uso que, en su caso, se le hubiera dado en el procedimiento. Se trata de una labor aparentemente sencilla, pero que en la práctica dista de serlo, porque exige tener en cuenta una multiplicidad de extremos de forma simultánea.

A la hora de llevar a cabo el examen global de una causa para evaluar el impacto que las infracciones procesales previas a la fase de juicio han podido tener en el derecho al proceso justo, desde el asunto Ibrahim el TEDH viene afirmando que se ha de tener en cuenta una compleja serie de factores, que no constituyen un catálogo cerrado.

Esos factores (que en buena medida compilan elementos ya ponderados en resoluciones anteriores) serían, entre otros, los siguientes: si el sujeto contra el que se dirigían las actuaciones es una persona especialmente vulnerable por razón de su edad o de su capacidad mental; si se cumplieron las previsiones legales internas relativas a la investigación preliminar y a la admisibilidad de las pruebas (pues si, pese a haberse producido una infracción grave, las pruebas resultantes han sido expurgadas de las actuaciones en aplicación de alguna regla de exclusión establecida por el derecho interno, el derecho al proceso justo no se habrá visto afectado); si el perjudicado tuvo oportunidad real de poner en cuestión la validez de las pruebas y oponerse a su uso; si las circunstancias en que se obtuvieron las pruebas arrojan dudas sobre su credibilidad intrínseca (particularmente, cuando se aplicó presión o coacción física o psicológica sobre el investigado); si las pruebas se obtuvieron de forma ilegal o con vulneración de algún otro de los derechos reconocidos en el $\mathrm{CEDH}$; tratándose de declaraciones, si se retractaron o modificaron con posterioridad, y en qué momento; si las pruebas en cuestión formaron parte o no del material probatorio de cargo que se utilizó para sustentar la condena, atendido el resto de pruebas practicadas; si la declaración de culpabilidad la hizo un tribunal profesional o un jurado lego; si existe un interés público especialmente intenso en la investigación y el castigo del concreto delito perseguido, $y$, finalmente, si en el supuesto examinado entraron en juego otras garantías procesales, previstas en la legislación o en la práctica procesal internas, que hayan podido compensar o mitigar de alguna manera los efectos de la infracción producida ${ }^{35}$.

De lo dicho hasta ahora se desprende que, en Ibrahim, el TEDH vino a establecer un nuevo test, destinado a reemplazar al del asunto Salduz. El test de la sentencia Ibrahim tiene también dos fases. En primer lugar, debe comprobarse la concurrencia de razones imperiosas para restringir el acceso temprano a un abogado. Tanto si las hubo como si no, debe examinarse a renglón

35 STEDH Ibrahim, $\$ 274$. 
seguido el posible impacto de la restricción en la equidad global de la causa, a la luz de las circunstancias que se acaban de enunciar. La diferencia entre ambas situaciones (existencia o inexistencia de razones de peso) estribaría en que, en los casos de falta de motivos suficientes para restringir el derecho de acceso temprano a un abogado, habría que aplicar un "escrutinio más estricto", que pasaría por arrojar sobre el Estado demandado la carga de alegar y probar de manera convincente que, aunque no se garantizó la debida asistencia letrada sin motivo aparente, pese a todo la equidad global del proceso no se vio perjudicada de forma irreparable ${ }^{36}$.

Importa mucho percatarse del sutil cambio de perspectiva que se produce en Ibrahim en cuanto a la segunda fase del control. El Tribunal parece abandonar por completo la óptica del derecho de defensa y la necesidad de preservarlo prospectivamente ya desde los primeros estadios de la investigación preliminar (un aspecto relativamente bien definible en términos de técnica jurídica), para adoptar un enfoque mucho más lato, de perfiles mucho más difusos y más difíciles de controlar, que es el de la equidad global (overall fairness) de la causa entendida como un todo.

A la vista quedan entonces la pluralidad, la diversidad y la complejidad de los elementos en juego, que llevan a que, a partir de Ibrahim, el criterio del TEDH ante supuestas vulneraciones del art. 6.3 del CEDH sea harto impredecible en la práctica; lo cual no es de extrañar del todo. Como se ha señalado antes, excepción hecha de las vulneraciones del art. 3 del CEDH, el Tribunal se ha mostrado siempre muy reacio a crear reglas de exclusión o prohibiciones de valoración de carácter estricto a partir de las previsiones del Convenio ${ }^{37}$; y parece encontrarse cómodo en una amplia zona gris de notable indefinición, que le permite resolver los distintos asuntos en función de las circunstancias particulares de cada caso concreto, atendiendo a consideraciones de justicia material, de interés público y — por qué no reconocerlo— también de oportunidad política.

\section{LA APLICACIÓN DEL ESTÁNDAR DE LA EQUIDAD GLOBAL DE LA CAUSA MÁS ALLÁ DE LA PERSECUCIÓN DE LOS ILÍCITOS MÁS GRAVES: ASUNTO SIMEONOVI C. BULGARIA}

Pese a la insistencia del TEDH en que existe continuidad de criterios en su jurisprudencia sobre esta materia, lo cierto es que la rectificación de rumbo

36 STEDH Ibrahim, $\$ 265$.

37 Sobre este particular, vid. Ölçer (2013: 371-399). Más recientemente, Bachmaier Winter (2019: 844-849). 
marcada por Ibrahim supone en la práctica una regresión a tiempos anteriores incluso a la sentencia John Murray ${ }^{38}$.

Esta impresión se corrobora a partir de la lectura de la sentencia de la Gran Sala en el asunto Simeonovi c. Bulgaria ${ }^{39}$, que se aparta definitivamente de la línea inaugurada en Salduz y consolida la aplicación de los criterios de la sentencia Ibrahim, extendiéndolos más allá de los supuestos de terrorismo.

La sentencia Simeonovi empieza afirmando que el derecho del art. 6.3 c) del $\mathrm{CEDH}$ no es un "derecho autónomo», sino que debe analizarse en el contexto del conjunto de garantías que aseguran la equidad del proceso tomado como un todo ${ }^{40}$. A renglón seguido, reproduce punto por punto la argumentación de Ibrahim: el derecho de acceso a un abogado es susceptible de restricción en sede policial si hay «razones imperiosas» para ello, pero la ausencia de esas razones no implica necesariamente que se haya producido una violación del art. 6 del $\mathrm{CEDH}$, porque lo verdaderamente importante es si la restricción (sea debida o indebida) ha lesionado la equidad global del proceso, para lo cual habrá que tener en cuenta, primordialmente, la lista de elementos de juicio compendiada en Ibrahim $^{41}$. La constatación de que, en un supuesto determinado, no había motivos que justificasen la privación de la asistencia letrada, tiene el efecto de desplazar sobre el Estado demandado la carga de mostrar de manera convincente que, pese a todo, la equidad global de la causa no se vio perjudicada de forma irreparable ${ }^{42}$. Se daría entonces en estos casos una suerte de "presunción de inequidad del proceso», que tocaría al Estado demandado despejar ${ }^{43}$.

Sobre la base de estas consideraciones, y tras realizar un recorrido por las vicisitudes de la tramitación del proceso en el asunto al que se refería la demanda, el TEDH llegó a la conclusión de que, en aquel supuesto, no se produjo vulneración alguna del art. 6 del Convenio — aunque sí de su art. 3.- Conviene

38 Vid., por ejemplo, la sentencia del asunto Imbrioscia, que data de 1993.

39 STEDH (GS) de 12 de mayo de 2017, asunto Simeonovi c. Bulgaria.

40 STEDH Simeonovi, $\$ 113$.

41 STEDH Simeonovi, $\$ 120$, que invoca expresamente el $\$ 274$ de la sentencia Ibrabim.

42 STEDH Simeonovi, $\$ \$ 116-118$. El Tribunal vuelve a insistir en que, en estos casos, debe aplicarse un «escrutinio muy estricto» en el examen de conjunto de las actuaciones, pero los términos en los que se concretaría ese examen reforzado quedan sin definir.

43 Esta conclusión aparece afirmada de forma expresa en el voto particular conjunto de los jueces Sajó, Lazarova-Trajkovska, Vučinić y Turković a la sentencia Simeonovi; y se ha incorporado a algún pronunciamiento posterior del TEDH, que habla de una "présomption de manque d'équité qui naît en l'absence de raisons impérieuses de retarder l'assistance juridique» («una presunción de falta de equidad que nace cuando no hay razones imperiosas para demorar la asistencia letrada»). $C f r$ la STEDH de 11 de diciembre de 2018, asunto Rodionov c. Rusia, $\$ 162$. 
destacar que el demandante, a quien se imputaban un delito de robo con violencia y dos asesinatos, no había tenido posibilidad de hablar con un abogado en los tres primeros días y medio de la detención, sin que se acreditaran (ni se adujeran siquiera) por parte de las autoridades «razones imperiosas» para imponer tal restricción. Como los primeros días de la detención no se habían documentado en forma alguna, no había manera de saber si era cierto que en ese tiempo el detenido había sido interrogado varias veces por la policía, como él afirmaba y el Estado búlgaro negaba. Desde el primer momento (y a lo largo de los casi cuatro meses en que estuvo privado de libertad), vivió encerrado en un calabozo sin ventanas, sin retrete y sin agua corriente, pobremente iluminado y sin posibilidad de aseo personal, con una sola cama pese a tratarse de una habitación compartida con otros dos individuos más. $\mathrm{Y}$, sin embargo, al no constar documentalmente en las actuaciones que se hubiera practicado ninguna otra diligencia ni que se hubiera extraído ninguna declaración autoincriminatoria del sujeto investigado en los primeros días de la detención, y sí haber constancia, en cambio, de una confesión realizada en presencia de un abogado dos semanas más tarde, así como de la regularidad del resto de actuaciones procesales posteriores, el TEDH desestimó la demanda en lo tocante a la denuncia de la infracción del art. 6 del CEDH, por entender que, pese a la injustificada privación inicial del derecho a la inmediata asistencia letrada, el proceso había sido «justo» contemplado en su conjunto.

Curiosamente, y contra el criterio manifestado en Ibrahim, en este caso el TEDH vino a desplazar de facto sobre el condenado la carga de alegar y acreditar la existencia de un nexo causal entre la falta de asesoramiento legal durante los primeros días de la detención y la confesión realizada dos semanas más tarde, y entre esta y el resto del material probatorio utilizado en su contra. Al no haberse efectuado ninguna alegación expresa en cuanto a la existencia de ese nexo, dio por sentado sencillamente que no lo había ${ }^{44}$.

\section{LA CONSOLIDACIÓN DE LA LÍNEA JURISPRUDENCIAL POST-IBRAHIM: ASUNTO BEUZE C. BÉLGICA}

La última resolución de la Gran Sala dictada hasta la fecha que reviste importancia en el tema que nos ocupa es la sentencia en el asunto Beuze c. Bélgica ${ }^{45}$.

44 Llevado a sus últimas consecuencias, el mensaje de Simeonovi es, desde luego, alarmante. Puede encontrarse una crítica extensa y fundamentada en los dos votos particulares que acompañan a la sentencia, que ponen de relieve las contradicciones que presenta con Salduz e Ibrahim.

45 STEDH (GS) de 9 de noviembre de 2018, asunto Beuze c. Bélgica. 
Por sus circunstancias, el asunto Beuze es en cierta medida similar al abordado por la sentencia Salduz. Se trata del caso de un ciudadano - en este caso mayor de edad- que, arrestado en Francia en virtud de una orden europea de detención, fue entregado a las autoridades belgas por el asesinato de su expareja. El sr. Beuze fue interrogado en Bélgica por la policía y por el instructor de la causa sin asistencia letrada. Tras acordarse la prisión provisional pudo designar un abogado, pero este no estuvo presente en los numerosos interrogatorios a los que fue sometido con posterioridad (un total de diez, entre los practicados por la policía, el juez de instrucción y el fiscal), ni tampoco en una reconstrucción de hechos que se llevó a cabo. En el transcurso de todas estas diligencias el sr. Beuze no se autoinculpó ni una sola vez, pero ofreció versiones diferentes de los hechos e incurrió en múltiples contradicciones. Celebrado el juicio, fue condenado por un jurado popular a la pena de cadena perpetua.

$\mathrm{Al}$ igual que las autoridades turcas en el asunto Salduz, las autoridades belgas actuaron en este caso con arreglo a la interpretación usual de la ley entonces vigente en el país, que admitía la práctica de las diligencias mencionadas sin la intervención del abogado del investigado. De hecho, este es precisamente uno de los puntos principales abordados por el Tribunal, puesto que, en su demanda, el sr. Beuze denunciaba que la legislación y la práctica belgas violaban por sí solas el art. $6.3 \mathrm{c}$ ) del Convenio, con independencia de su aplicación en cualesquiera supuestos concretos.

Tras reiterar las proclamaciones anteriores del TEDH sobre el derecho de acceso temprano a un abogado, la sentencia Beuze comienza afirmando que el mero hecho de designar un abogado no garantiza suficientemente por sí solo la asistencia jurídica efectiva al detenido; sino que, para cumplir con las exigencias del art. 6 del CEDH, es preciso respetar una serie de mínimos ${ }^{46}$. Así, debe asegurarse la posibilidad de contacto con el abogado desde que el sujeto pasa a estar bajo custodia policial; debe permitírsele consultar con él antes de cualquier interrogatorio (e incluso si no lo hay), y el abogado tiene que poder entrevistarse con su defendido en privado y recibir instrucciones confidenciales. Además, el letrado designado tiene que poder estar presente durante los interrogatorios, tanto policiales como a cargo de otras autoridades. Durante el curso de estos, debe brindársele la posibilidad de prestar al interrogado un asesoramiento real y efectivo, no meramente formal, así como permitírsele velar por que el derecho de defensa no se vea indebidamente comprometido merced a la conducta de su defendido. Por último, a la hora de valorar el carácter real y efectivo de la asistencia prestada desde el

46 STEDH Beuze, $\$ 132$. 
punto de vista de la equidad global del proceso, deben examinarse caso por caso todos los extremos normalmente asociados a la intervención de la figura del abogado, como pueden ser la discusión del caso, la organización de la defensa, el acopio de material exculpatorio, la preparación para los interrogatorios, el apoyo en situaciones de conmoción o turbación (distress) y la verificación de las condiciones de la detención ${ }^{47}$.

Por lo que hace a la relación entre la justificación de la restricción del derecho y la equidad global del proceso, la sentencia Beuze viene a distinguir dos tipos de situaciones. La primera se produce cuando la limitación del acceso temprano a un abogado está prevista con carácter general y sistemático en una ley, como en los asuntos John Murray, Salduz o el mismo asunto Beuze. La segunda situación se da cuando se está ante una restricción ad hoc, inducida por circunstancias peculiares y excepcionales de un caso particular, como sucedió en el asunto $\mathrm{Ibrahim}^{48}$. En Beuze, la Gran Sala reconoce de forma explícita que alguna vez ha habido secciones del TEDH que directamente han entendido - casi siempre en relación con Turquía- que una restricción sistemática ex lege del derecho a acceder a un abogado vulnera sin más el art. 6 del $\mathrm{CEDH}^{49}$. Pero, al mismo tiempo, recuerda que hay un buen número de sentencias que se apartan de ese enfoque, efectuando un control en la línea de Salduz, es decir, entrando pese a todo a ponderar tanto la posible concurrencia de razones imperiosas para restringir el derecho en el caso concreto como, en su caso, la equidad del proceso considerado en su conjunto. La propia Gran Sala se habría decantado por este segundo criterio de manera definitiva en Ibrahim $^{50}$.

Esto sentado, la sentencia Beuze trae a colación el criterio fijado en Salduz, conforme al cual la existencia de una previsión legal que permita limitar con carácter general el acceso temprano a un abogado no constituye una «razón imperiosa» en el sentido exigido por la jurisprudencia del TEDH. Pero de inmediato advierte, como ya había advertido en Ibrahim, que al examinar este punto la sentencia Salduz no se detuvo en la

47 STEDH Beuze, $\$ 136$. La enunciación de todos estos extremos — cuyo valor no es otro, por el momento, que el de simples exhortaciones obiter dicta - proviene originariamente de la STEDH en el asunto Dayanan c. Turquía.

48 STEDH Beuze, $\$ 139$.

49 Cita concretamente a título de ejemplo la sentencia Dayanan y la STEDH de 9 de febrero de 2010, asunto Boz c. Turquía, si bien hay otras muchas, entre las que cabría mencionar, en relación con un ordenamiento distinto del turco, la STEDH de 12 de enero de 2016, asunto Borg c. Malta.

50 STEDH Beuze, \$\$ 139-141. 
constatación de la ausencia de razones para restringir el acceso al abogado, sino que siguió analizando en términos de equidad global de la causa las consecuencias de recibir como prueba las declaraciones realizadas en esas circunstancias $^{51}$.

Habida cuenta de la importancia del derecho al acceso temprano a un abogado, tres son los requisitos que, según Beuze, deben cumplirse para que su restricción resulte legítima desde el punto de vista de las exigencias del Convenio: que se trate de una limitación temporal, que tenga carácter excepcional y que se base en un análisis particularizado de las circunstancias del caso. También cuando hay previsión legal que faculta (o incluso obliga) a adoptarla, porque haya previsión legal o no, las autoridades nacionales tienen que justificar que concurren razones imperiosas que aconsejan restringir el acceso del detenido a un abogado en el caso concreto ${ }^{52}$. Como se afirmó en Ibrahim — que se atuvo en este punto a la redacción del art. 3.6 de la Directiva 2013/48/UE_, es razón imperiosa a estos efectos, por ejemplo, una necesidad urgente de evitar graves perjuicios (serious adverse consequences; une atteinte grave) para la vida, la libertad o la integridad física de alguien ${ }^{53}$.

Sea como fuere, el TEDH hace notar que Ibrahim afirmó de forma inequívoca que la ausencia de razones imperiosas para restringir el derecho no entraña por sí sola una vulneración del art. 6.3 c) del Convenio. Tanto si se ha justificado la concurrencia de esas razones como si no, hay que examinar la causa entera a fin de ponderar si, contemplada en su conjunto, ha satisfecho los estándares del proceso justo pese a la limitación inicial del acceso a un abogado. La Sala viene a reiterar así que no hay aquí regla de exclusión automática alguna, como parecía desprenderse de pronunciamientos posteriores a Salduz de alguna sección del Tribunal ${ }^{54}$. Según Beuze, la aplicación del estándar de la justicia del proceso en su conjunto se ajusta a la aproximación que efectúa siempre la jurisprudencia de Estrasburgo para el resto de posibles vulneraciones del art. 6 del CEDH; aproximación que, al mismo tiempo, resultaría coherente con el papel del TEDH, que no consistiría en "armonizar legislaciones» ni en «emitir juicios en abstracto», sino en establecer salvaguardias para asegurarse de que los procedimientos seguidos en cada caso respetan las exigencias del juicio justo, de acuerdo con

\footnotetext{
$51 \quad$ STEDH Beuze, $\$ 138$.

52 STEDH Beuze, $\$ 142$.

53 STEDH Ibrahim, $\$ \$ 210$ y 259.

54 STEDH Beuze, 144. Menciona expresamente la sentencia Dayanan y «otras resoluciones contra Turquía».
} 
las circunstancias de cada acusado 55 . A la hora de hacer el examen de conjunto del proceso a efectos de determinar el impacto de las posibles irregularidades iniciales en su «equidad global», el Tribunal reitera que hay que atender a la lista de parámetros de control enunciados en el parágrafo 274 de la sentencia Ibrahim $^{56}$.

Hechas las consideraciones que preceden, el Tribunal entró a conocer del fondo del asunto planteado en la demanda. A tal efecto, constató que, en relación con el sr. Beuze, se había producido una restricción patente del derecho de acceso a un abogado; que la restricción se había basado en disposiciones de carácter general, aplicadas mecánicamente por las autoridades belgas sin atender a las circunstancias específicas de su caso, y que no se habían alegado ni acreditado por el Estado afectado razones imperiosas de ningún tipo para acordarla. A continuación, siguiendo la pauta de Ibrahim, se detuvo a comprobar si la equidad global de la causa se había visto afectada negativamente por la restricción del derecho a la asistencia letrada temprana. A este respecto, valoró diversos extremos: el sujeto había declarado en comisaría; sus declaraciones fueron detalladas y contradictorias; fueron recibidas en el juicio a efectos probatorios sin que el jurado entrase a valorar las circunstancias en las que se habían producido, ni tampoco el posible impacto de la falta de asesoramiento jurídico en su realización; desempeñaron un importante papel en la vertebración de la acusación, y constituyeron la prueba de cargo en relación con uno de los delitos imputados (una tentativa de homicidio, cuya autoría quedó, además, establecida a partir de las manifestaciones del detenido). Por otro lado, los miembros del jurado no fueron convenientemente instruidos acerca de si debían valorarlas, y cómo. Para Beuze, fue el juego combinado de todos estos factores lo que provocó que el proceso en su conjunto no fuera justo en el sentido exigido por el art. 6 del CEDH, habiéndose producido una vulneración de la letra c) de su apartado tercero.

En resoluciones posteriores, el TEDH se ha venido ateniendo a estos principios con regularidad ${ }^{57}$.

55 STEDH Beuze, $\$ \$ 147-149$. Estos dos parágrafos acogen en esencia la tesis expresada en el voto particular conjunto de los jueces Spano y Lemmens a la STEDH de 18 de noviembre de 2014, asunto Aras c. Turquía (2).

56 STEDH Beuze, $\$ 150$.

57 Véanse las SSTEDH de 11 de diciembre de 2018, asunto Rodionov c. Rusia; de 24 de enero de 2019, asunto Knox c. Italia; de 5 de febrero de 2019, asunto Utvenko y Borisov c. Rusia; de 19 de febrero de 2019, asunto Ruşen Bayar c. Turquía; de 16 de abril de 2019, asunto Alakhverdyan c. Ucrania; de 23 de mayo de 2019, asunto Doyle 


\section{CONSIDERACIONES FINALES}

Las cinco resoluciones examinadas evidencian que, a lo largo de los veinticinco últimos años, se ha producido una evolución muy notable de la jurisprudencia de Estrasburgo en lo que toca a la legitimidad, desde el punto de vista de las exigencias del art. 6 del Convenio, de las restricciones al derecho a la asistencia letrada en las diligencias policiales. Pueden distinguirse tres fases en esa evolución. La primera abarca el período que media entre la sentencia John Murray y la sentencia Salduz (de 1996 a 2008). La segunda comprende los ocho años que separan Salduz de Ibrahim (2016). La tercera fase - en la que todavía nos encontramos en el momento de escribirse estas líneas- sería la que parte de la sentencia Ibrahim; y últimamente se ha visto refrendada en sus aspectos esenciales por los pronunciamientos recaídos en Simeonovi (2017) y Beuze (2018).

Todo parece indicar que los cambios no han terminado aún, y que el momento actual es un momento de transición de duración incierta. Sin embargo, la tesitura en la que nos encontramos dista de ser idónea; y así se ha puesto de manifiesto con argumentos de peso desde dentro del propio Tribuna ${ }^{58}$. Como se decía al principio de este trabajo, da la impresión de que, en la actualidad, el examen de toda denuncia relativa a cualquiera de las garantías del art. 6.3 del CEDH se termina reconduciendo por el TEDH a una comprobación de si la causa judicial fue o no justa en términos globales ${ }^{59}$. Desde luego, esta "aproximación holística» constituye una manera habilidosa de asegurarse un amplio margen de discernimiento, que permite al Tribunal utilizar varas de medir distintas en función de las circunstancias (dependiendo, por ejemplo, de la clase de procedimiento que se haya seguido, de la gravedad de los hechos o del país del que provenga la demanda). Pero es a la postre problemática, en la medida en que, llevada a sus últimas consecuencias, entraña una relativización de esas garantías, que quedan privadas de sustantividad y significación propias.

En efecto, si se parte de la base de que la conculcación injustificada de cualquiera de ellas en el caso concreto no determina por sí sola una violación

c. Irlanda; de 28 de mayo de 2019, asunto Van de Kolk c. Holanda, y de 4 de junio de 2019, asunto Farrugia c. Malta.

58 Vid., por ejemplo, el voto particular del juez Serghides a la sentencia Simeonovi; el voto particular de los jueces Yudkivska, Vučinić, Turković y Hüseynov a la sentencia Beuze, o el del juez Pinto de Alburquerque a la STEDH (GS) de 18 de diciembre de 2018, asunto Murtazaliyeva c. Rusia (\$\$ 37-46).

59 En el pasado no siempre fue así. Al respecto, vid. Goss (2017: 1142-1145). 
del Convenio, porque lo verdaderamente decisivo es el impacto que tal conculcación haya podido tener en la probidad o equidad del proceso tomado como un todo, la conclusión que cabe extraer es que el apartado $3 .^{\circ}$ del art. 6 del $\mathrm{CEDH}$ carece de eficacia inmediata real, pues las garantías concretas que enumera poseen una función meramente orientativa o ejemplificadora. Son simples pautas de control, que han de ser tenidas en cuenta al analizar el caso concreto, pero nada más. No habría entonces diferencia sustancial a efectos prácticos entre las "garantías-pauta» del art. 6.3 del $\mathrm{CEDH}$ y los factores de ponderación que aparecen listados en los parágrafos 274 de la sentencia Ibrahim y 150 de la sentencia Beuze. Toda la virtualidad del art. 6 del CEDH se agotaría en un único elemento, la rúbrica, y el examen de toda posible denuncia relativa a este se resolvería en definitiva en verificar la equidad o integridad jurídica (fairness) del proceso con una mirada omniabarcante.

Ciertamente, como ha declarado de manera reiterada el TEDH, los derechos del art. 6.3 del Convenio no son fines en sí mismos. Su carácter es eminentemente instrumental, pues todos ellos se ordenan a asegurar a su vez el derecho a un proceso justo. Sin embargo, el concepto de «proceso justo" no debería ser (o no debería configurarse jurisprudencialmente como) una categoría borrosa de exclusiva apreciación casuística, precisamente porque el Convenio se ha preocupado de identificar y enunciar una serie de parámetros objetivos, cuyo respeto en principio constituiría, salvo excepciones debidamente justificadas - u oportunamente remediadas o compensadas-, el mínimo formal exigible a priori a toda causa penal que pretenda ser tenida por justa. Así resulta, de hecho, de la dicción literal del primer inciso del mismo art. 6.3 del CEDH. Desde esta óptica, la fundamentación de Salduz es desde luego más consistente que las tesis alternativas que terminaron por prevalecer en decisiones posteriores del Tribunal (siempre y cuando se parta de la base, claro está, de que el concepto de "cargos penales» del art. 6.3 del CEDH es, en efecto, un concepto autónomo, que autoriza para proyectar las garantías que ese precepto consagra sobre las pesquisas o actuaciones previas al proceso) ${ }^{60}$.

No se quiere postular con esto en absoluto el definitivo abandono del llamado overall fairness test. Al contrario, el examen del proceso bajo el prisma de su equidad en términos globales resulta indispensable cuando se trata de esclarecer si una limitación o privación razonada de cualquiera de las garantías del art. 6.3 del CEDH menoscaba pese a todo el derecho a un proceso justo; o para dilucidar si un cúmulo de irregularidades menores ha dado lugar a una lesión de

60 El carácter automático de la aplicación de este presupuesto ha sido puesto en cuestión. Vid. al respecto el voto particular del juez Mahoney a la sentencia Ibrahim. 
ese derecho ${ }^{61}$. Y, por supuesto, se revela como un instrumento enormemente útil a la hora de abordar determinadas cuestiones respecto de las cuales el Convenio guarda silencio, y en las que una actitud maximalista o un intervencionismo directo por parte del Tribunal podrían hacer peligrar gravemente su auctoritas $^{62}$.

Un caso paradigmático es el de la espinosa cuestión de si el derecho a un proceso justo del art. 6 del $\mathrm{CEDH}$ exige que la información obtenida con vulneración de otros derechos reconocidos por el Convenio deba necesariamente verse afectada por una exclusión probatoria o no ${ }^{63}$. A falta de una regla de exclusión expresa en el texto del CEDH, y a falta también de una uniformidad de criterio en los distintos ordenamientos estatales, dar entrada por vía jurisprudencial a una medida de esta índole de alcance general sería probablemente percibido en todas partes como una extralimitación de los jueces de Estrasburgo. Ello aconseja manejarse con cautela, que es lo que, en efecto, ha venido haciendo desde siempre el TEDH. Sin dar carta de naturaleza en ningún momento a la vieja máxima male captum, bene retentum, procura mostrar sensibilidad hacia las circunstancias particulares de cada caso, para lo cual el análisis de conjunto de las actuaciones se ha revelado como un expediente sumamente valioso, dotado de una muy oportuna maleabilidad.

Ahora bien, a la luz de las consideraciones que preceden, cabe cuestionar la conveniencia de trasladar este expediente también al examen de denuncias relativas a la privación injustificada de derechos y garantías que el Convenio sí enuncia de forma expresa, que aparecen además configurados en él como auténticos mínimos impuestos por el derecho a un proceso justo (y sin las puntualizaciones, dicho sea de paso, que otros preceptos del CEDH efectúan respecto de los derechos que a su vez consagran $\left.{ }^{64}\right)^{65}$.

Sea como fuere, importa mucho advertir que las oscilaciones jurisprudenciales que se han descrito a lo largo de estas páginas no son un fenómeno aislado, sino que forman parte de un contexto más amplio, que es el de una

${ }^{61}$ Cfr. la STEDH de 6 de diciembre de 1988, asunto Barberà, Messegué y Jabardo c. España, \$89.

62 Para una perspectiva más amplia sobre el overall fairness test, pueden consultarse Goss (2014: 124-139) y Weisser (2019: 93-95).

63 En general, sobre el tema, vid. Ölçer (2013). En particular sobre las declaraciones autoinculpatorias realizadas sin presencia letrada, vid. Giannoulopoulos (2019: 165-199).

${ }^{64} \mathrm{Cfr}$., por ejemplo, las salvedades que introducen los arts. 8.2 y $10.2 \mathrm{del} \mathrm{CEDH.}$

65 En el mismo sentido, vid. Goss (2017: 1149-1150). A ello se refería recientemente también el juez Pinto de Alburquerque, criticando con dureza lo que califica de «expansión corrosiva» del overall fairness test. Vid. su extenso voto particular a la STEDH (GS) de 18 de diciembre de 2018, asunto Murtazaliyeva c. Rusia. 
cierta crisis institucional del TEDH, inmerso desde hace tiempo en un debate doctrinal y político sobre la necesidad de acometer una reforma de calado ${ }^{66}$. Después de unos años de intensa actividad judicial en ámbitos diversos, que ha podido deteriorar su imagen institucional en determinados países ${ }^{67}$, el Tribunal parece haber decidido moderar el paso, mostrándose dispuesto a observar un mayor nivel de deferencia en la revisión de la actuación de las autoridades nacionales ${ }^{68}$. En este sentido, es significativo que el arrumbamiento de la prometedora línea inaugurada en Salduz se haya producido precisamente a raíz de un supuesto como el abordado en el asunto Ibrahim c. Reino Unido ${ }^{69}$. Es más, si se tiene en cuenta lo dicho hasta ahora, no parece adecuado limitarse a valorar las implicaciones de esta decisión en estrictos términos técnicos de garantismo versus eficacia (es decir, desde el lugar común de la tensión que habitualmente existe entre las garantías procesales y la eficaz persecución de los crímenes más graves, como pueden ser los delitos de terrorismo), pues resulta obvio que entraron aquí en juego también consideraciones de "política judicial» que, dado su peso decisivo en la posición finalmente adoptada por el Tribunal, no deben pasarse por alto.

Pese al posterior cambio de rumbo de Estrasburgo, Salduz no fue para nada un pronunciamiento infecundo. Como se ha visto, incidió de modo determinante en el ordenamiento interno de varios países, desencadenando cambios normativos de enorme trascendencia. Además, ejerció un influjo crucial en los trabajos preparatorios de la Directiva 2013/48/UE (algún autor ha llegado a referirse a ella como «la Directiva $S a l d u z »)^{70}$, que, sin el precedente

66 Vid. al respecto las declaraciones resultantes de las conferencias intergubernamentales de Interlaken (2010), Izmir (2011), Brighton (2012), Bruselas (2015) y Copenhague (2018).

67 Puede encontrarse una exposición panorámica relativamente completa en los trabajos reunidos en Popelier, Lambrecht y Lemmens (2016). Sobre el caso del Reino Unido, por su especial enjundia doctrinal, vid. Finnis (2016) y Malcolm (2017).

68 Sobre el estándar deferencial del «margen de apreciación» en la jurisprudencia de Estrasburgo y su carácter problemático, vid. García Roca (2010). Para un estudio analítico -y crítico - sobre su empleo por el TEDH en los últimos años, vid. Gerards (2018).

69 Conviene tener presente el contexto temporal de la sentencia Ibrahim: uno de los puntos de debate en la campańa electoral de 2015 en el Reino Unido fue justamente el de si el país debía abandonar el Consejo de Europa; discusión que quedó en cierto modo eclipsada a raíz del referéndum sobre el Brexit del ańo siguiente. Al respecto, vid. Amos (2017: 764-765).

$70 C f r$. Spronken (2012). Para una sucinta visión panorámica de la situación anterior a Salduz en el derecho comparado, vid. Jackson (2016: 993-1000). 
de aquella sentencia del TEDH, probablemente habría salido adelante con un contenido muy diferente al que tiene ahora. Puede decirse, de hecho, que los principios de Salduz perviven todavía en cierta forma a través de esta directiva europea, pues figuran insertos de algún modo en el texto finalmente adop$\operatorname{tado}^{71}$. Al mismo tiempo, conviene aclarar que la Directiva 2013/48/UE no llegó a imponer de forma inequívoca y directa la introducción en los ordenamientos internos de una regla de exclusión de las declaraciones obtenidas con vulneración del derecho a un abogado. En este punto, el legislador europeo optó por remitirse «a las disposiciones y los sistemas nacionales en materia de admisibilidad de pruebas» (art. 12.2 de la directiva); aunque precisando que, en la valoración de las declaraciones así conseguidas (allí donde esté prevista), los Estados miembros deben asegurar «el respeto a los derechos de defensa y las garantías de un juicio justo». No cabe duda del importante potencial transformador que posee este inciso, para cuya efectiva actualización habrá que esperar, llegado el caso, a que tenga ocasión de pronunciarse el TJUE.

En último término, los problemas que se le han planteado a Estrasburgo con la garantía del art. 6.3 c) del CEDH obedecen a que, en el Convenio (concretamente en el art. 5), falta una disposición equivalente al inciso final del art. 17.3 de la Constitución española. Esta circunstancia constrińe al Tribunal Europeo de Derechos Humanos a llevar a cabo una interpretación extensiva un tanto forzada del art. $6.3 \mathrm{del} \mathrm{CEDH}$, a fin de poder proyectarlo sobre las diligencias policiales. Al mismo tiempo, como los derechos que enumera ese artículo se conciben como garantías del proceso justo, no hay manera de despejar definitivamente la sombra de una posible relativización de corte casuístico; pues mientras estemos hablando del art. 6 del CEDH, la apreciación de una violación del Convenio por irregularidades cometidas en sede policial siempre se podrá hacer depender, en última instancia, de que el derecho a un proceso justo se haya visto efectivamente comprometido desde el punto de vista material. Porque lo cierto es que el Convenio no contempla en ninguno de sus preceptos un derecho a la asistencia letrada que goce de autonomía funcional respecto del derecho a un proceso equitativo.

Salduz fue un audaz intento, a la postre fallido, de romper con esta dinámica. Pretendió introducir un elemento formal de control —el estándar de las «razones imperiosas»—, de modo que hubiese que entender que la privación

71 Para una propuesta de pautas generales de interpretación de las previsiones de la directiva a partir de Salduz, vid. Pivaty (2018). Sobre las posibles divergencias entre el nivel de protección que la directiva obliga a asegurar a los Estados miembros y los criterios adoptados en la jurisprudencia de Estrasburgo a partir de Ibrahim, vid. Soo (2017). 
inmotivada del acceso a un abogado en dependencias policiales determina de suyo una automática vulneración del derecho a un proceso justo, sin más matizaciones ni más distinciones. La decisión adoptada en Salduz se fundaba en un razonamiento lineal: el art. 6.3 del Convenio establece los mínimos exigibles a un proceso justo. Es así que, con arreglo a la jurisprudencia anterior, las garantías del proceso justo deben extenderse a las actuaciones policiales, aunque la causa penal no esté iniciada en sentido estricto. Ergo, en sede policial deben respetarse imperativamente los mínimos del art. 6.3 del $\mathrm{CEDH}$, salvo que concurran razones especiales que justifiquen su limitación en un caso concreto y así se acredite oportunamente por las autoridades.

Sin embargo, en retrospectiva se aprecia con claridad que, en aquel momento, faltaba todavía la convicción ampliamente compartida que por lo común necesita un cambio de semejante trascendencia para resultar irreversible, tanto entre los Estados como dentro del propio Tribunal. En el fondo, por positiva que sea la valoración que deba merecer Salduz, seguramente le estaba pidiendo al art. 6.3 c) del CEDH más de lo que podía dar entonces (y, muy posiblemente, más de lo que podría dar también ahora). Quién sabe si, en un futuro no muy lejano, la situación será otra.

\section{Bibliografía}

Amos, M. (2017). The value of the European Court of Human Rights to the United Kingdom. European Journal of International Law, 28 (3), 763-785. Disponible en: https://doi.org/10.1093/ejil/chx044.

Anagnostopoulos, I. (2014). The Right of Access to a Lawyer in Europe: A Long Road Ahead? European Criminal Law Review, 4, 3-18. Disponible en: https://doi.org/10.5235/ 219174414811783333.

Arangüena Fanego, C. (2014). El derecho a la asistencia letrada en la Directiva 2013/48/UE. Revista General de Derecho Europeo, 32, 1-31.

Bachmaier Winter, L. (2015). The EU Directive on the Right to Access to a Lawyer: a Critical Assessment. En S. Ruggeri (ed.). Human Rights in European Criminal Law: New Developments in European Legislation and Case Law after the Lisbon Treaty (pp. 111-131). Heidelberg: Springer. Disponible en: https://doi.org/10.1007/978-3-319-12042-3_7. (2019). Rights and Methods to Challenge Evidence and Witnesses in Civil Law Jurisdictions. En D. Brown, J. Turner y B. Weisser (eds.). The Oxford Handbook of Criminal Process (pp. 841-864). Oxford: Oxford University Press.

Beernaert, M. A., Charrière-Bournazel, C. y Jeanneret, Y (2012). Les suites de l'arrêt Salduz en droit belge, français et Suisse. Revue Trimestrielle des Droits de l'Homme, 23 (90), 241-269.

Buric, Z. (2018). Re-assessing the Jurisprudence of the European Court of Human Rights on Police Interrogation - Case of Ibrahim and Others v. The United Kingdom. En 
D. Duic y T. Petrasevic (eds.). EU Law in Context - Adjustment to Membership and Challenges of the Enlargement (pp. 338-354). Osijek: JJS University of Osijek. Disponible en: https://doi.org/10.25234/eclic/7116.

Celiksoy, E. (2018). Ibrahim and Others v. UK: Watering down the Salduz principles? New Journal of European Criminal Law, 9, 229-246. Disponible en: https:/doi.org/ $10.1177 / 2032284418778149$.

Cras, S. (2014). The Directive on the right of access to a lawyer in criminal proceedings and in European arrest warrant proceedings. Eucrim, 1, 32-44. Disponible en: https://doi. org/10.30709/eucrim-2014-005.

Finnis, J. (2016). Judicial Law-Making and the Living Instrumentalisation of the ECHR. En R. Ekins, P. Yowell, y N. Barber (eds.). Lord Sumption and the Limits of the Law (pp. 73-120). Oxford: Hart Publishing.

García Roca, J. (2010). El margen de apreciación nacional en la interpretación del Convenio Europeo de Derechos Humanos: soberanía e integración. Madrid: Civitas-Thomson.

Gerards, J. (2018). Margin of Appreciation and Incrementalism in the Case Law of the European Court of Human Rights. Human Rights Law Review, 18, 495-515. Disponible en: https://doi.org/10.1093/hrlr/ngy017.

Giannoulopoulos, D. (2016). Strasbourg Jurisprudence, Law Reform and Comparative Law: A Tale of the Right to Custodial Legal Assistance in Five Countries. Human Rights Law Review, 16, 103-129. Disponible en: https://doi.org/10.1093/hrlr/ngv039.

- (2019). Improperly Obtained Evidence in Anglo-American and Continental Law. Oxford: Hart Publishing.

Goss, R. (2014). Criminal Fair Trial Rights. Article 6 of the European Convention on Human Rights. Oxford: Hart Publishing.

- (2017). Out of Many, One? Strasbourg's Ibrahim Decision on Article 6. The Modern Law Review, 80, 1137-1163. Disponible en: https://doi.org/10.1111/1468-2230.12305.

Jackson, J. D. (2016). Responses to Salduz: Procedural Tradition, Change and the Need for Effective Defence. The Modern Law Review, 79, 987-1018. Disponible en: https://doi. org/10.1111/1468-2230.12227.

Jimeno Bulnes, M. (2014). La Directiva 2013/48/UE del Parlamento europeo y del Consejo de 22 de octubre de 2013 sobre los derechos de asistencia letrada y comunicación en el proceso penal: ¿realidad al fin? Revista de Derecho Comunitario Europeo, 48, 443-489.

Maffei, S. y Sonenshein, D. (2012). The Cloak of the Law and Fruits Falling from the Poisonous Tree: A European Perspective on the Exclusionary Rule in the Gäfgen Case. Columbia Journal of European Law, 19, 21-55.

Malcolm, N. (2017). Human Rights and Political Wrongs. London: Policy Exchange.

Mols, V. (2017). Bringing Directives on procedural rights of the EU to police stations: practical training for criminal defence lawyers. New Journal of European Criminal Law, 8, 300-308. Disponible en: https://doi.org/10.1177/2032284417723421.

Ölçer, F. P. (2013). The European Court of Human Rights: The Fair Trial Analysis Under Article 6 of the European Convention of Human Rights. En S. C. Thaman (ed.). Exclusionary Rules in Comparative Law (pp. 371-399). Heidelberg: Springer. Disponible en: https://doi.org/10.1007/978-94-007-5348-8_16.

Ormazábal Sánchez, G. (2015). El derecho a no autoincriminarse. Madrid: Civitas. 
Pivaty, A. (2018). The Right to Custodial Legal Assistance in Europe: In Search for the Rationales. European Journal of Crime, Criminal Law and Criminal Justice, 26, 62-98. Disponible en: https://doi.org/10.1163/15718174-02601004.

Popelier, P., Lambrecht, S. y Lemmens, K. (2016). Criticism of the European Court of Human Rights. Shifting the Convention System: Counter-dynamics at the National and EU Level. Cambridge: Intersentia. Disponible en: https://doi.org/10.1017/9781780685175.

Soo, A. (2017). Divergence of European Union and Strasbourg Standards on Defence Rights in Criminal Proceedings? Ibrahim and Others $v$. the UK (13th of September 2016). European Journal of Crime, Criminal Law and Criminal Justice, 25, 327-346. Disponible en: https://doi.org/10.1163/15718174-02504002.

Spronken, T. (2012). Effective Defence: The Letter of Rights and the Salduz-Directive. En G. Vermeulen (ed.). Defence Rights: International and European Developments (pp. 81-102). Antwerp: Maklu.

Weisselberg, C. D. (2017). Exporting and Importing Miranda. Boston University Law Review, 97, 1235-1291.

Weisser, B. (2019). The European Convention on Human Rights and the European Court of Human Rights as guardians of fair criminal proceedings in Europe. En D. K. Brown, J. I. Turner y B. Weisser (eds.). The Oxford Handbook of Criminal Procedure, (pp. 89-114). Oxford: Oxford University Press. Disponible en: https://doi.org/10.1093/ oxfordhb/9780190659837.013.6. 